\title{
Postpartum blood loss: visual estimation versus objective quantification with a novel birthing drape
}

\author{
Tripop Lertbunnaphong ${ }^{1}$, MD, Numporn Lapthanapat ${ }^{1}$, BNS, Jarunee Leetheeragul ${ }^{1}$, BNS, APN, \\ Pussara Hakularb ${ }^{1}$, BNS, MA, Amporn Ownon $^{1}$, BSc
}

INTRODUCTION Immediate postpartum haemorrhage (PPH) is the most common cause of maternal mortality worldwide. Most recommendations focus on its prevention and management. Visual estimation of blood loss is widely used for the early detection of PPH, but the most appropriate method remains unclear. This study aimed to compare the efficacy of visual estimation and objective measurement using a sterile under-buttock drape, to determine the volume of postpartum blood loss.

METHODS This study evaluated patients aged $\geq 18$ years with low-risk term pregnancies, who delivered vaginally. Immediately after delivery, a birth attendant inserted the drape under the patient's buttocks. Postpartum blood loss was measured by visual estimation and then compared with objective measurement using the drape. All participants received standard intra- and postpartum care.

RESULTS In total, 286 patients with term pregnancies were enrolled. There was a significant difference in postpartum blood loss between visual estimation and objective measurement using the under-buttock drape $(178.6 \pm 133.1 \mathrm{~mL}$ vs. $259.0 \pm 174.9 \mathrm{~mL} ; \mathrm{p}<0.0001$ ). Regarding accuracy at $100 \mathrm{~mL}$ discrete categories of postpartum blood loss, visual estimation was found to be inaccurate, resulting in underestimation, with low correspondence (27.6\%) and poor agreement (Cohen's kappa coefficient $0.07 ; p<0.05$ ), compared with objective measurement using the drape. Two-thirds of cases of immediate PPH (65.4\%) were misdiagnosed using visual estimation.

CONCLUSION Visual estimation is not optimal for measurement of postpartum blood loss in PPH. This method should be withdrawn from standard obstetric practice and replaced with objective measurement using the sterile under-buttock drape.

Keywords: postpartum blood loss, postpartum haemorrhage, sterile under-buttock drape, visual estimation

\section{INTRODUCTION}

Immediate postpartum haemorrhage $(\mathrm{PPH})$ is defined as blood loss $\geq 500 \mathrm{~mL}$ after normal vaginal delivery. ${ }^{(1-3)}$ This obstetric complication is a leading cause of maternal death worldwide. ${ }^{(2)}$ To prevent the occurrence of $\mathrm{PPH}$, current evidence supports active management of the third stage of labour, especially the prescription of oxytocin, as standard obstetric care. ${ }^{(1-3)}$ However, the optimal method for determining the volume of postpartum blood loss for early detection of PPH remains unclear.

Although the ideal measurement of postpartum blood loss involves the use of objective tools, visual estimation is still commonly used in daily obstetric practice. ${ }^{(4)}$ Photospectometry is the gold standard blood loss measurement technique due to its accuracy. However, this technique is complicated, costly and impractical. Additionally, photospectrometry cannot be applied at all levels of healthcare and is more suitable for clinical research. ${ }^{(4,5)}$ In a previous study, an alternative method weighed soaked swabs or drapes after delivery, which proved to be effective for the early detection of PPH. ${ }^{(6)}$ However, this method substantially increases the workload of medical staff members and may not be suitable in a busy hospital setting.

The use of sterile under-buttock or postpartum drapes is another method of postpartum blood loss measurement. This method is suitable for low-resource hospitals, as it is inexpensive, convenient and easy to use. Previous studies have shown that it produced results that highly correlated with those of standard photospectrometry (correlation coefficient $[r]=0.93$ ), with a reduction in postpartum haemoglobin levels reported..$^{(5,7,8)}$ Therefore, this method was chosen as the gold standard in the present study.

The purpose of this study was to compare the efficacy of visual estimation and objective measurement using the sterile under-buttock drape to determine the volume of postpartum blood loss in PPH. We hope that the results of this study will lead to improvements in standard obstetric care for the early detection of immediate $\mathrm{PPH}$.

\section{METHODS}

With the ethical approval of the Siriraj Institutional Review Board, the present study was prospectively conducted from June 2013 to December 2013. Pregnant Thai women aged $\geq 18$ years who were admitted in the early phase of labour were included in the study. Women who had painful contractions, were near delivery, or were under the sedative effect of morphine or its derivatives were excluded. This study also excluded women who had bleeding disorders (e.g. idiopathic thrombocytopenic purpura), dependence on bleeding-related medication (e.g. aspirin), a Caesarean delivery, fetal anomalies or a stillbirth. All the 
women provided written informed consent prior to admission to the labour room and were managed according to our standard intrapartum protocol. Immediately after normal vaginal delivery, 20 units of oxytocin in 1,000 mL normal saline were delivered intravenously at $120 \mathrm{~mL} / \mathrm{hr}$, combined with a slow intravenous injection of methylergometrine $0.2 \mathrm{mg}$ as standard medical prevention of immediate PPH.

The sterile under-buttock drape used in this study was a single-use drape made from a large, clear plastic bag measuring $35.6 \mathrm{~cm} \times 55.9 \mathrm{~cm}$ and costing less than USD 1 per bag. The bag was modified to serve as a pouch by cutting its upper edge (Fig. 1). Immediately after delivery of the baby, a birth attendant inserted this drape under the buttocks of the mother. Consequently, the placenta was delivered via modified Credé manoeuvre or controlled cord traction, depending on the experience of the birth attendant. Uterine massage was performed for all patients. Blood from placental delivery and perineorrhaphy was collected in the pouch. On completion of perineorrhaphy, the healthcare providers who delivered the baby, including doctors, nurses and medical students, visually estimated the volume (in $\mathrm{mL}$ ) of postpartum blood loss. Subsequently, the blood from the plastic pouch was poured into a standard cylinder and measured. The total blood loss by visual estimation was then compared with that objectively measured using the sterile under-buttock drape. Patients with immediate PPH underwent care based on the standard protocol for management of immediate $\mathrm{PPH}$.

All data was collected and analysed with PASW Statistics version 18.0 for Windows (SPSS Inc, Chicago, IL, USA). The characteristics of the study population were evaluated by descriptive analysis and reported either as mean \pm standard deviation or number (percentage). The difference in postpartum blood loss volume between visual estimation and objective measurement with the sterile under-buttock drape was analysed using dependent Student's $t$-test. Agreement between methods was analysed using Cohen's kappa coefficient. The accuracy rate of visual estimation compared to objective measurement with the sterile under-buttock drape was also evaluated. A p-value $<0.05$ was considered statistically significant.

\section{RESULTS}

In total, 286 participants met the inclusion criteria and were enrolled in the study. The mean age of the participants was $27.1 \pm 5.9$ years and the majority were nulliparous $(46.2 \%)$. The pregnancy outcomes are shown in Table I. The mean durations of the first, second and third stages of labour were normal. Most patients delivered with the assistance of doctors (85.3\%), without anaesthesia $(68.2 \%)$ or without instrument-assisted delivery (96.5\%). Most newborns were of normal weight (i.e. 2,500-4,000 g; $88.8 \%$ ). No pregnancies had serious complications that required surgical intervention or blood transfusion. None of the newborns exhibited severe birth asphyxia.

There was a significant difference $(80.4 \pm 41.8 \mathrm{~mL} ; \mathrm{p}<0.0001)$ in the overall volume of blood loss between visual estimation (mean $178.6 \pm 133.1 \mathrm{~mL}$ ) and objective measurement with the

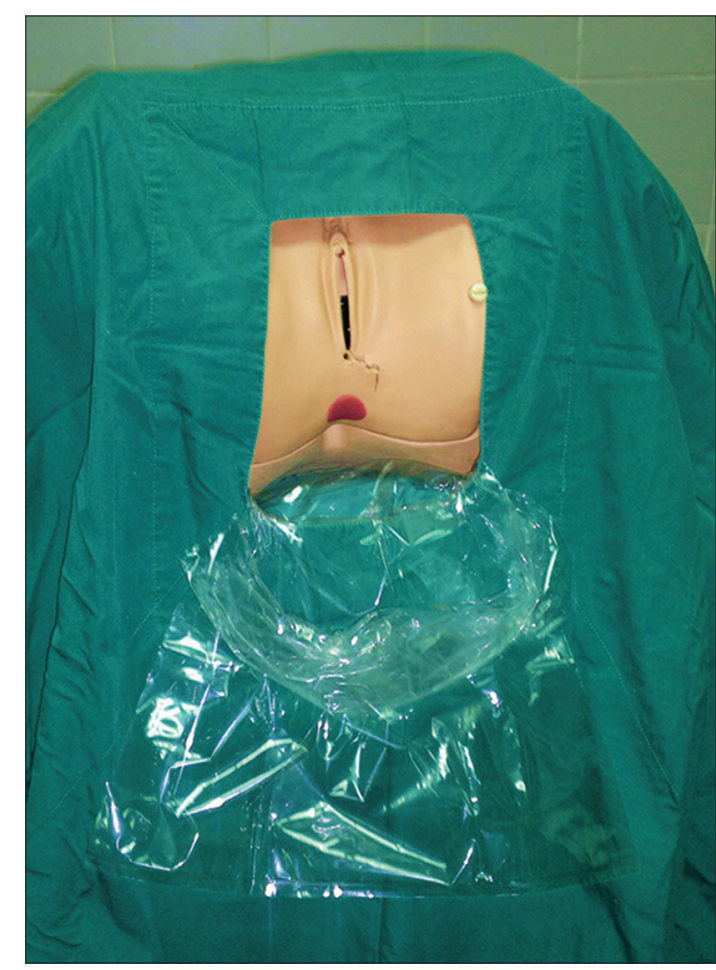

Fig. 1 Photograph shows the sterile under-buttock drape, made from a large, clear plastic bag.

Table I. Pregnancy outcomes of study population $(n=286)$.

\begin{tabular}{|c|c|}
\hline Outcome & No. (\%) \\
\hline \multicolumn{2}{|l|}{ Duration of labour (min) } \\
\hline First stage & $522.2 \pm 282.6^{*}$ \\
\hline Second stage & $28.9 \pm 27.5^{*}$ \\
\hline Third stage & $7.4 \pm 3.6^{*}$ \\
\hline \multicolumn{2}{|l|}{ Obstetric anaesthesia } \\
\hline None & $195(68.2)$ \\
\hline Intravenous meperidine & $88(30.8)$ \\
\hline Epidural anaesthesia & $3(1.0)$ \\
\hline \multicolumn{2}{|l|}{ Mode of delivery } \\
\hline Normal vaginal delivery & $276(96.5)$ \\
\hline Vacuum extraction & $8(2.8)$ \\
\hline Forceps extraction & $2(0.7)$ \\
\hline \multicolumn{2}{|l|}{ Birth attendant } \\
\hline Doctor & $244(85.3)$ \\
\hline Nurse & $32(11.2)$ \\
\hline Medical student & $10(3.5)$ \\
\hline Fetal birth weight (g) & $3,012.3 \pm 413.6^{*}$ \\
\hline$<2,500$ & $31(10.8)$ \\
\hline$\geq 2,500-4,000$ & $254(88.8)$ \\
\hline$>4,000$ & $1(0.3)$ \\
\hline \multicolumn{2}{|l|}{ Birth asphyxia } \\
\hline None & $272(95.1)$ \\
\hline Mild & $8(2.8)$ \\
\hline Moderate & $6(2.1)$ \\
\hline
\end{tabular}

*Data presented as mean \pm standard deviation.

sterile under-buttock drape (mean $259.0 \pm 174.9 \mathrm{~mL}$ ). Visual estimation of postpartum blood loss was around $31 \%$ less accurate than objective measurement with the sterile under-buttock drape. 
Table II. Accuracy and underestimation using visual estimation at $100 \mathrm{~mL}$ discrete categories of postpartum blood loss ( $\mathbf{n}=\mathbf{2 8 6}$ ).

\begin{tabular}{|c|c|c|c|c|c|c|c|c|}
\hline \multirow{2}{*}{$\begin{array}{l}\text { Sterile under-buttock } \\
\text { drape measurement }(\mathrm{mL})\end{array}$} & \multicolumn{6}{|c|}{ Visual estimation $(\mathrm{mL})$} & \multirow{2}{*}{$\begin{array}{c}\text { Accuracy } \\
\text { (\%) }\end{array}$} & \multirow{2}{*}{$\begin{array}{c}\text { Underestimation } \\
\text { (\%) }\end{array}$} \\
\hline & $0-100$ & $101-200$ & $201-300$ & $301-400$ & 401-499 & $\geq 500$ & & \\
\hline $0-100$ & 21 & 6 & 0 & 0 & 0 & 0 & 77.8 & 0 \\
\hline $101-200$ & 67 & 35 & 2 & 0 & 0 & 0 & 33.7 & 64.4 \\
\hline $201-300$ & 21 & 45 & 9 & 1 & 0 & 0 & 11.8 & 86.8 \\
\hline $301-400$ & 3 & 20 & 14 & 5 & 0 & 1 & 11.6 & 86.0 \\
\hline $401-499$ & 0 & 5 & 2 & 3 & 0 & 0 & 0 & 100 \\
\hline$\geq 500$ & 0 & 2 & 9 & 5 & 1 & 9 & 34.6 & 65.4 \\
\hline
\end{tabular}

Data presented as no. of participants, unless otherwise stated.

Table II compares the accuracy of visual estimation versus objective measurement with the sterile under-buttock drape at $100 \mathrm{~mL}$ discrete categories of postpartum blood loss. The incidence of immediate $\mathrm{PPH}$, as assessed by visual estimation and objective measurement with the sterile under-buttock drape, was $3.5 \%$ and $9.1 \%$, respectively, with uterine atony being the most common cause of PPH. Comparatively, visual estimation had poor agreement (Cohen's kappa coefficient 0.07; $\mathrm{p}<0.05$ ) and low correspondence (27.6\%) rates. In each category, after $100 \mathrm{~mL}$ of blood loss, visual estimation also showed an underestimation of blood loss in the majority of cases. Overall, only $34.6 \%$ of patients were diagnosed with immediate $\mathrm{PPH}$.

\section{DISCUSSION}

Visual estimation of postpartum blood loss, a simple and convenient method, has been a routine practice for a long time, even though some researchers have claimed that this method is inaccurate and results in the underestimation of the actual volume of blood loss. ${ }^{(4-7,9)}$ Simulation training to improve visual estimation has not resulted in an improvement in the quality of measurement. Error, low accuracy and unreliability remain, especially in cases of massive haemorrhage. ${ }^{(10-13)}$ Previous studies have reported that, independent of the experience or skill level of healthcare providers, visual estimation of postpartum blood loss has a $25 \%-89 \%$ error of measurement. ${ }^{(5,6,9,14)}$ The present study supports these findings. Similarly, a study by Prasertcharoensuk et $\mathrm{al}^{(9)}$ reported error of measurement using visual estimation as compared with direct measurement; however, details of the direct measurement protocol was not explained. In addition, the incidence of immediate PPH was relatively high (up to $27.63 \%$ ) and seemed to be an overexpectation. The present study clearly described the objective measurement protocol using the sterile under-buttock drape. The $9.1 \%$ incidence of immediate PPH in the present study was three times lower than that of Prasertcharoensuk et al's study.

Visual estimation is an unreliable tool for the measurement of postpartum blood loss. In previous studies, the error of visual estimation was much higher in patients with a high postpartum blood loss volume and much lower in patients with a low postpartum blood loss volume..$^{(9,11,15)}$ However, the present study showed different findings. Compared with objective measurement with the under-buttock drape, visual estimation resulted in underestimation at all $100 \mathrm{~mL}$ discrete categories of blood loss. The incidence of underestimation using visual estimation was over $50 \%$, with very low accuracy, a low correspondence rate $(27.6 \%)$ and poor agreement (Cohen's kappa coefficient $0.07 ; p<0.05$ ). Furthermore, it resulted in misdiagnosis of $\mathrm{PPH}$ in one patient who had a low volume of blood loss, which led to unnecessary intervention and wastage of resources. Two-thirds of immediate PPH (65.4\%) was missed using this subjective method. Therefore, we found that visual estimation is a low-quality and inaccurate method that frequently leads to underestimation at all discrete categories of blood loss. Although none of the patients had any adverse outcomes in the present study, it can be assumed that visual estimation could lead to delayed diagnosis and is thus not an appropriate tool for the early detection of immediate $\mathrm{PPH}$.

Objective measurement, especially using a postpartum calibrated drape or bag, is an appropriate method for determining postpartum blood loss for early diagnosis of immediate PPH. In 2011, Al Kadri et al ${ }^{(6)}$ reported the use of a gravimetric method, or weighing of blood collected in all delivery materials on a sensitive scale, to diagnose immediate $\mathrm{PPH}$. The authors reported that this method was superior to visual estimation, which had a blood loss measurement error of about 30\%. ${ }^{\left({ }^{6}\right)}$ Although the present study had similar findings to Al Kadri et al's study, our objectives and results differ. While Al Kadri et al's study aimed to compare the accuracy of the gravimetric method against visual estimation of postpartum blood loss by professionals (i.e. doctors and nurses), our study compared the error of visual estimation against a better measurement tool (i.e. the sterile under-buttock drape). Although the gravimetric method is superior to visual estimation, it is complicated and time-consuming to use, especially in a busy clinical setting; this drawback was likewise acknowledged by the authors. ${ }^{(6)}$ The sterile under-buttock drape, on the other hand, is a better method for measuring postpartum blood loss due to its relative simplicity and ease of use. Significant postpartum bleeding usually results from the episiotomy wound and poor contraction of the uterine myometrium after placental delivery, which causes bleeding from blood vessels at the placental implantation site. ${ }^{(16)}$ To avoid contamination with amniotic fluid, we recommend that the drape be used immediately after delivery of the baby and before delivery of the placenta. Furthermore, the present study also examined the accuracy of visual estimation at $100 \mathrm{~mL}$ discrete categories of postpartum blood loss and confirmed significant errors in this method.

$\mathrm{Al}$ Kadri et al's study reported that the actual incidence of $\mathrm{PPH}$ in Saudi Arabia was very low, at only $1.47 \%$; ${ }^{(6)}$ although 
this study involved a small sample size and was conducted in a developing country, it is possible that the incidence could have been underestimated. In the present study, which involved a larger number of participants than the aforementioned study, the incidence of $\mathrm{PPH}$, as diagnosed using visual estimation of blood loss volume, was $3.5 \%$, while that of objective measurement using the sterile under-buttock drape was $9.1 \%$. Our finding is similar to that of Tourné et al, ${ }^{(17)}$ who reported a $10 \%$ incidence of $\mathrm{PPH}$ with the use of a collecting bag for measurement of blood loss volume, further supporting the utility of the under-buttock drape as a rapid, low-cost tool for measuring blood loss in $\mathrm{PPH}$.

In 2010, Zhang et $\mathrm{al}^{(18)}$ reported that the use of a collection bag did not reduce the rate of severe $\mathrm{PPH}$. This result, however, has yet to be fully investigated. The study, which involved many developed countries, did not define a standard care protocol for $\mathrm{PPH}$ among these countries. The study also included Caesarean delivery, for which it is difficult to accurately measure the postpartum blood loss volume. ${ }^{(6)}$ Therefore, the method used in Zhang et al's study cannot be applied to all clinical settings, especially in developing or low-resource countries.

In 2011, Tixier et al ${ }^{(7)}$ prospectively compared 122 patients' postpartum blood loss volume using a combination of underbuttock drapes and collection pouches, with measurement of changes in haemoglobin and haematocrit levels. They found a $14.75 \%$ incidence of immediate $\mathrm{PPH}$ and concluded that the collection pouch was highly sensitive in the early diagnosis of immediate PPH, especially if postpartum blood loss volume had reached $300 \mathrm{~mL} .{ }^{(7)}$ Tixier et al's finding is supported by Patel et al, ${ }^{(5)}$ who used the BRASS-V blood collection drape as the gold standard for measuring PPH volume, due to its high correlation with standard photospectrometry $(r=0.93)$. Their study likewise concluded that the blood collecting bag was useful, valuable and significantly more accurate than visual estimation of blood loss. ${ }^{(5)}$

The strength of the present study is the prospective nature of the trial, which involved a larger number of participants than prior studies ${ }^{(5-7,9)}$ and produced highly reliable data. Postpartum blood loss was recorded by visual estimation among birth attendants of various skill levels; additionally, sterile under-buttock drape measurement, which was performed by another care provider, was highly accurate based on standardised objective measurements. Therefore, data collection bias was not present in this study. Furthermore, the study focused mainly on the value of visual estimation and provided clear evidence that this method has little value and lacks accuracy in determining the volume of postpartum blood loss and diagnosing PPH.

A weakness of this study was that only the blood in the underbuttock drape, but not the blood soaked in gauze packs or swabs, was measured. Hence, the actual incidence of immediate PPH may have been higher than the current reported incidence. It would have been better if a combined approach, i.e. measuring the blood loss volume in the drape as well as weighing all gauze packs or swabs, had been used; the latter could be performed using a simple weighing scale in the delivery suite. The sum of these two methods should provide a better estimation of postpartum blood loss.

In conclusion, the present study illustrates that visual estimation of postpartum blood loss is inaccurate, resulting in underestimation and misdiagnosis of immediate $\mathrm{PPH}$. Therefore, we propose that visual estimation of postpartum blood loss be withdrawn from standard obstetric practice and replaced with a more appropriate method, such as objective measurement using a sterile under-buttock drape.

\section{REFERENCES}

1. World Health Organization. WHO recommendations for the prevention and treatment of postpartum haemorrhage. Geneva: WHO Press, 2012.

2. Lalonde A; International Federation of Gynecology and Obstetrics. Prevention and treatment of postpartum hemorrhage in low-resource settings. Int J Gynaecol Obstet 2012; 117:108-18.

3. American College of Obstetricians and Gynecologists. ACOG Practice Bulletin: Clinical Management Guidelines for Obstetrician-Gynecologists Number 76, October 2006: postpartum hemorrhage. Obstet Gynecol 2006; 108:1039-47.

4. Schorn MN. Measurement of blood loss: review of the literature. J Midwifery Womens Health 2010; 55:20-7.

5. Patel A, Goudar SS, Geller SE, et al. Drape estimation vs. visual assessment for estimating postpartum hemorrhage. Int J Gynaecol Obstet 2006; 93:220-4

6. Al Kadri HM, Al Anazi BK, Tamim HM. Visual estimation versus gravimetric measurement of postpartum blood loss: a prospective cohort study. Arch Gynecol Obstet 2011; 283:1207-13.

7. Tixier H, Boucard C, Ferdynus C, Douvier S, Sagot P. Interest of using an underbuttocks drape with collection pouch for early diagnosis of postpartum hemorrhage. Arch Gynecol Obstet 2011; 283:25-9.

8. Toledo P, McCarthy RJ, Hewlett BJ, Fitzgerald PC, Wong CA. The accuracy of blood loss estimation after simulated vaginal delivery. Anesth Analg 2007; 105:1736-40.

9. Prasertcharoensuk W, Swadpanich U, Lumbiganon P. Accuracy of the blood loss estimation in the third stage of labor. Int J Gynaecol Obstet 2000; 71:69-70.

10. Maslovitz S, Barkai G, Lessing JB, Ziv A, Many A. Improved accuracy of postpartum blood loss estimation as assessed by simulation. Acta Obstet Gynecol Scand 2008; 87:929-34.

11. Buckland SS, Homer CS. Estimating blood loss after birth: using simulated clinical examples. Women Birth 2007; 20:85-8.

12. Sukprasert M, Choktanasiri W, Ayudhya NI, Promsonthi P, O-Prasertsawat P. Increase accuracy of visual estimation of blood loss from education programme. J Med Assoc Thai 2006; 89 Suppl 4:S54-9.

13. Toledo P, McCarthy RJ, Burke CA, et al. The effect of live and web-based education on the accuracy of blood-loss estimation in simulated obstetric scenarios. Am J Obstet Gynecol 2010; 202:400.e1-5.

14. Yoong W, Karavolos S, Damodaram M, et al. Observer accuracy and reproducibility of visual estimation of blood loss in obstetrics: how accurate and consistent are health-care professionals? Arch Gynecol Obstet 2010; 281:207-13

15. Glover P. Blood loss at delivery: how accurate is your estimation? Aust J Midwifery 2003; 16:21-4

16. Cunningham FG, Leveno KJ, Bloom S, et al. Obstetrical hemorrhage. In: Williams Obstetrics. 24th ed. New York: McGraw-Hill Education, 2014: 780-828.

17. Tourné G, Collet F, Lasnier P, Seffert P. [Usefulness of a collecting bag for the diagnosis of post-partum hemorrhage]. J Gynecol Obstet Biol Reprod (Paris) 2004; 33:229-34. French.

18. Zhang WH, Deneux-Tharaux C, Brocklehurst P, et al; EUPHRATES Group. Effect of a collector bag for measurement of postpartum blood loss after vaginal delivery: cluster randomised trial in 13 European countries. BMJ 2010; 340:c293. 